\title{
Highly efficient and eco-friendly one-pot synthesis of penta substitute pyrrole derivatives under catalyst-free conditions
}

\author{
Denish J. Viradiya*, Bharat H. Baria, Rajesh Kakadiya, Vipul C. Kotadiya, \\ Anamik Shah** \\ Department of Chemistry (UGC-SAP \& DST-FIST Sponsored), Saurashtra University, \\ Rajkot - 360005, Gujarat, India
}

National Facility for Drug Discovery Center, Saurashtra University, Rajkot - 360005, Gujarat, India

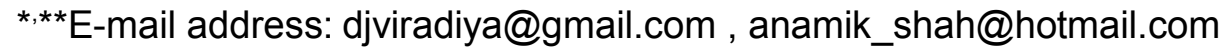

\begin{abstract}
An eco-friendly and efficient one-pot synthesis of penta-substituted pyrrole derivatives via a four-component reaction of maldrum's acid, arylglyoxal monohydrate, dimethyl but-2-yne-dioate and amines under catalyst-free conditions in an environmentally friendly medium is described. The simple experimental procedure, catalyst-free reaction conditions, short period of conversion, and excellent yields are the advantages of the present method. Good chemical yields have been achieved without the need for chromatography and recrystallization or other purification methods.
\end{abstract}

Keywords: One-pot synthesis; penta-substituted pyrrole derivatives; multi-component reactions

\section{INTRODUCTION}

The development of a simple, green reaction protocol for the synthesis bioactive medicinal motifs is an attractive area of research in both academia and the pharmaceutical industry. One of the most attractive approaches is to use of multi-component reactions (MCRs) using three or more starting materials in one-pot procedure to give a final complex product [1]. In the past decade, there have been tremendous developments in MCRs methodology and significant efforts continue to be made to develop new MCRs. Among the many heterocyclic compounds, the pyrrole ring has found a large number of applications and is present in many natural products [2]. It is used as an important skeleton in organic synthesis and is also utilized in other important fields, such as functional materials [3], medicinal chemistry and pharmacological agents. Up to date, many synthetic methods have been developed for the construction of pyrroles, including the classical Knorr reaction, the Hantzsch reaction [4], Buchwald-Hartwig coupling [5] and the Paal-Knorr condensation reaction. Additionally, other new methodologies, such as transition-metal catalyzed cyclizations [6], cyclo-addition reactions [7] and MCRs were developed for preparing new pyrrole derivatives. However; some of these methods have significant limits such as boring 
workup procedures, harsh reaction conditions, low yields, long reaction times or the condition for an inert atmosphere. Therefore, a simple and efficient method for pyrrole synthesis remains an attractive goal. On the other side, 2-substituted maldrum's acid, derivatives are of much importance because they exist in many natural products and exhibit a wide range of biological activities such as antibacterial, antiviral [8], anticoagulant, anti HIV, anticancer [9] and antioxidant activities [10]. A molecular scaffold which combines both maldrum's acid, and pyrrole moieties might integrate the properties of both, and the hybridization of the both heterocyclic systems in a single nucleus may result in the formation of some valuable molecules from a biological viewpoint. To the best of our knowledge, there have been no reports of synthesis of maldrum's acid fused pyrrole derivatives by multicomponent reactions. As a part of our ongoing research on the development of multicomponent approaches to heterocycles, herein we are reporting new synthesis of pentasubstituted pyrrole derivatives using MCRs.

\section{RESULT AND DISCUSSION}

We at first evaluated the four-component reaction of a $1: 1: 1: 1$ mixture of maldrum's acid (1), phenylglyoxal monohydrate (2), dimethyl but-2-ynedioate (3) and p-toluidine (4) under a variety of conditions (Scheme 1). The results are summarized in Table 1 . To find the best reaction conditions, we evaluated several different parameters including reaction temperature, solvent, and additives. Firstly, the synthesis of target compound (5a) was performed in a variety of solvent like acetone, DMF, THF, ethyl acetate, water, and ethanol. To our delight, all the reactions gave moderate to excellent yield of the desired product except DMF, and ethayl acetate. The best results were obtained when ethanol was used as a solvent at reflux and the reaction proceeded excellent affording the desired product in $83 \%$ yield within $1 \mathrm{~h}$ (Table 1, entry 7). Recently L-proline has been used as a catalyst for some multicomponent reactions. To optimize the reaction conditions and investigated the role of catalyst, L-proline was selected as the catalyst to promote this reaction. However, the use of L-proline as a catalyst showed no improvement in yield (Table 1, entry 8).

The optimized reaction conditions were then applied for the construction of library with maldrum's acid along with barbituric acid (1,3-dicarbonyl compounds), 4-F-phenylglyoxal monohydrates, dimethyl but-2-ynedioate, and six arometic amine. The corresponding pentasubstituted pyrrole derivatives 5(b-1) were obtained refluxing ethanol under catalyst free conditions. The result showed that the reaction is seen to be tolerant of substitution on the aniline ring with electron-donating groups at $\mathrm{C} 4$ - the position (Table 2). However, electronwithdrawing group present at the $\mathrm{C} 4$ position of aniline ring yielded compound with low yield (Table 2, entries 6 and12).

\section{1. Experimental Procedure}

\section{1. 1. Materials and methods}

${ }^{1} \mathrm{H}$ NMR spectra were recorded on a Bruker AV 3000 supercon (400 MHz) spectrometer using the deuterated solvent as an internal deuterium lock. Chemical shift data are given in units $\delta$ relative to residual protic solvent where $\delta\left(\mathrm{CDCl}_{3}-d\right)=7.24 \mathrm{ppm}$ and $\delta$ $\left(\right.$ DMSO- $\left.\mathrm{d}_{6}\right)=2.50 \mathrm{ppm}$. The multiplicity of a signal is indicated as: br-broad, s-singlet, $\mathrm{d}-$ doublet, t-triplet, q-quartet, m-multiplet etc. Coupling constant $(J)$ are quoted in $\mathrm{Hz}$ and recorded to the nearest $0.1 \mathrm{~Hz} .{ }^{13} \mathrm{C}$ NMR Spectra were recorded on a Bruker AV 3000 
supercon $(100 \mathrm{MHz})$ spectrometer with broadband proton decoupling using the deuterated solvent as an internal deuterium lock. Chemical shift data are given in units $\delta$ relative to residual protic solvent where $\delta\left(\mathrm{CDCl}_{3}-d\right)=77.23 \mathrm{ppm} \delta\left(\mathrm{DMSO}-d_{6}\right)=39.51 \mathrm{ppm}$.

\section{1. 2. Reaction Scheme}<smiles>CC1(C)OC(=O)CC(=O)O1</smiles><smiles>O=C1CC(=O)NC(=O)C1</smiles><smiles>O=C(c1ccc(F)cc1)C(O)O</smiles><smiles>[R]c1ccc(N)cc1</smiles>
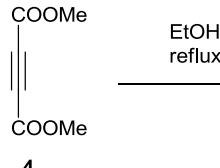
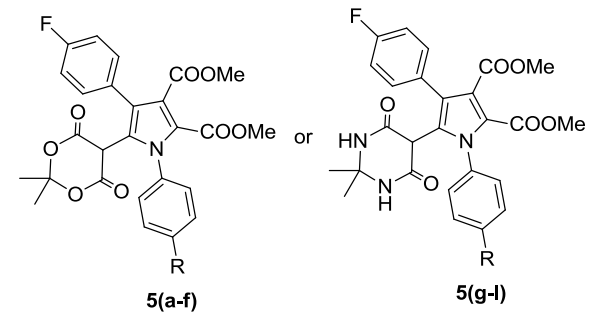

\section{1. 3. General procedure for the synthesis penta-substituted pyrrole}

A dry $25 \mathrm{~mL}$ flask was charged with maldrum's acid 1a $(1 \mathrm{mmol}), 4-F-p h e n y l g l y o x a l$ monohydrate 2 (1 mmol) or barbituric acic $1 \mathbf{b}(1 \mathrm{mmol})$, substituted anilines 3a-f $(1 \mathrm{mmol})$, dimethyl but-2-ynedioate $4(1 \mathrm{mmol})$, and ethanol $(5 \mathrm{~mL})$. The mixture was stirred at refluxing temperature for indicated time. After the completion of the reaction (confirmed by TLC), the reaction mixture was cooled to room temperature. The crude product obtained was finally purified by recrystallization from chloroform. The isolated compounds were characterized by IR, ${ }^{1} \mathrm{H} \mathrm{NMR},{ }^{13} \mathrm{C} \mathrm{NMR}$ and elemental analysis $(\mathrm{C}, \mathrm{H}$ and $\mathrm{N})$.

Table 1. Optimization of the reaction conditions for the synthesis of compound (5a).

\begin{tabular}{|c|c|c|c|c|c|}
\hline Entry & Solvent & $\begin{array}{c}\text { Temperature } \\
\left({ }^{\circ} \mathbf{C}\right)\end{array}$ & Additive & Time (h) & $\begin{array}{c}\text { Yield } \\
(\mathbf{\%})\end{array}$ \\
\hline 1 & DMF & 80 & - & 1 & 22 \\
\hline 2 & Acetone & 60 & - & 1 & 56 \\
\hline 3 & $\begin{array}{c}\text { Ethyl } \\
\text { acetate }\end{array}$ & 80 & - & 1 & 12 \\
\hline 4 & $\mathrm{H}_{2} \mathrm{O}$ & 80 & - & 1 & 45 \\
\hline 5 & $\mathrm{THF}$ & 80 & - & 1 & 64 \\
\hline 6 & EtOH & r.t. & - & 24 & 42 \\
\hline 7 & EtOH & 80 & - & 1 & 83 \\
\hline 8 & EtOH & 80 & L-proline & 1 & 81 \\
\hline
\end{tabular}

${ }^{\mathrm{a}}$ Reaction conditions:1a (1 mmol), 2 (1 mmol), 3 (1 mmol), 4 (1 mmol), solvent (5 mL). ${ }^{\mathrm{b}}$ Isolated yield. 
Table 2. The synthesis of penta-substituted pyrrole derivatives 5(a-1) via four-component reaction.

\begin{tabular}{|c|c|c|c|c|c|}
\hline Entry & $\begin{array}{l}\text { 1,3-dicarbonyl } \\
\text { compounds }\end{array}$ & $\mathbf{R}^{1}$ & Time (h) & Product & Yield (\%) \\
\hline 1 & & 4- $\mathrm{CH}_{3} \mathrm{C}_{6} \mathrm{H}_{4}$ & 1.0 & $5 a$ & 83 \\
\hline 2 & & $4-\mathrm{Cl} \mathrm{C} \mathrm{H}_{6} \mathrm{H}_{4}$ & 3.0 & $5 b$ & 76 \\
\hline 3 & & $\begin{array}{c}4-\mathrm{OCH}_{3} \\
\mathrm{C}_{6} \mathrm{H}_{4} \\
\end{array}$ & 1.2 & $5 c$ & 87 \\
\hline 4 & & $4-\mathrm{F} \mathrm{C}_{6} \mathrm{H}_{4}$ & 2.5 & $5 d$ & 72 \\
\hline 5 & & 4- $\mathrm{Br} \mathrm{C}_{6} \mathrm{H}_{4}$ & 2.7 & $5 e$ & 76 \\
\hline 6 & & $4-\mathrm{NO}_{2} \mathrm{C}_{6} \mathrm{H}_{4}$ & 4.2 & $5 f$ & 57 \\
\hline 7 & & $4-\mathrm{CH}_{3} \mathrm{C}_{6} \mathrm{H}_{4}$ & 1.0 & $5 \mathrm{~g}$ & 86 \\
\hline 8 & & 4- $\mathrm{Cl} \mathrm{C} \mathrm{C}_{6} \mathrm{H}_{4}$ & 2.7 & $5 \mathrm{~h}$ & 74 \\
\hline 9 & & $\begin{array}{c}4-\mathrm{OCH}_{3} \\
\mathrm{C}_{6} \mathrm{H}_{4} \\
\end{array}$ & 1.2 & $5 \mathrm{i}$ & 88 \\
\hline 10 & & $4-\mathrm{F} \mathrm{C}_{6} \mathrm{H}_{4}$ & 2.3 & $5 \mathrm{j}$ & 71 \\
\hline 11 & & 4- $\mathrm{Br} \mathrm{C}_{6} \mathrm{H}_{4}$ & 2.5 & $5 \mathrm{k}$ & 72 \\
\hline 12 & & $4-\mathrm{NO}_{2} \mathrm{C}_{6} \mathrm{H}_{4}$ & 3.8 & 51 & 52 \\
\hline
\end{tabular}

\section{1. 4. Proposed mechanism for the formation of compounds}

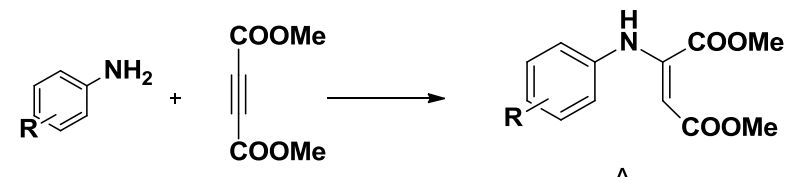

3

4
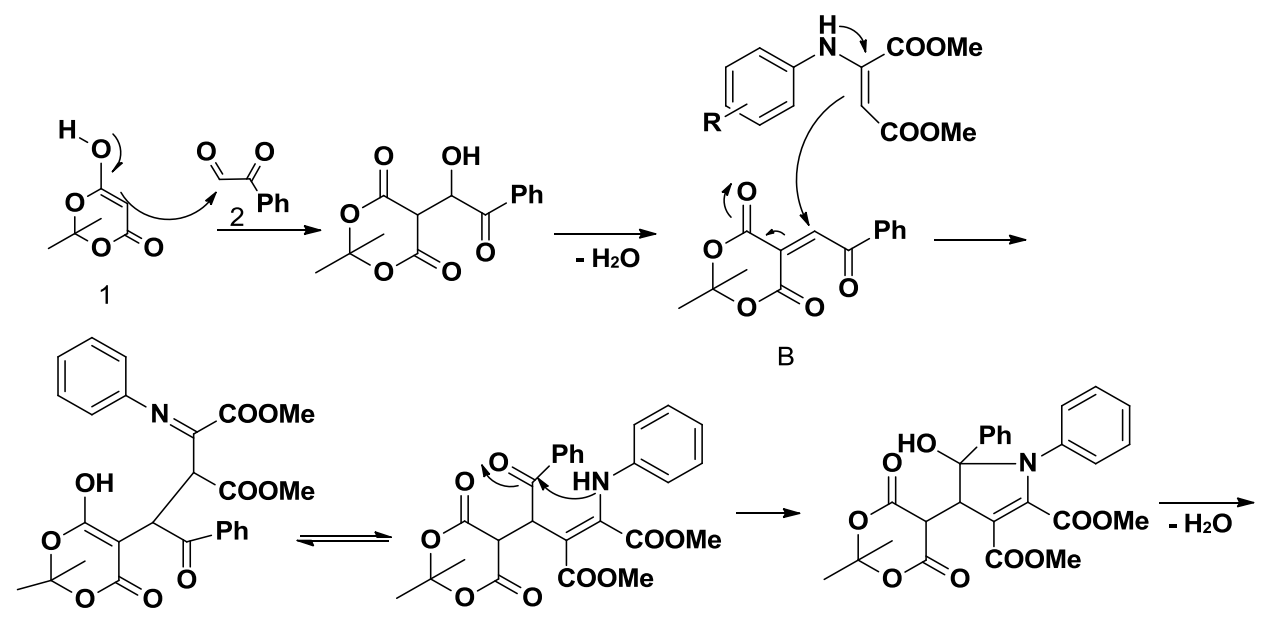

B

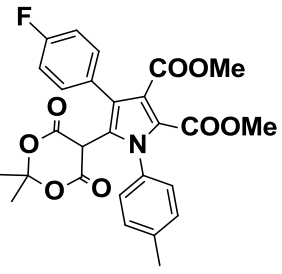

C

D 


\section{1. 5. Spectral data of the compounds}

Dimethyl,5-(2,2-dimethyl-4,6-dioxo-1,3-dioxan-5-yl)-4-(4-fluorophenyl)-1-(p-tolyl)-1Hpyrrole-2,3-dicarboxylate (5a)

Characteristic: white crystalline solid M.P.: $146-150{ }^{\circ} \mathrm{C}$; IR (KBr): 3032, 2845, 1691, 1622 , 1528, 1394, 1274, 1179, $753 \mathrm{~cm}^{-1}$; ${ }^{1} \mathrm{H}$ NMR (400 MHz, $\left.\mathrm{CDCl}_{3}\right): \delta 1.76\left(\mathrm{~s}, 6 \mathrm{H}, \mathrm{C}\left(\mathrm{CH}_{3}\right)_{2}\right)$, $2.27\left(\mathrm{~s}, 3 \mathrm{H},-\mathrm{CH}_{3}\right), 3.65\left(\mathrm{~s}, 6 \mathrm{H}\left(-\mathrm{COOCH}_{3}\right)\right), 5.01(\mathrm{~s}, 1 \mathrm{H}-\mathrm{CH}), 7.05(\mathrm{~d}, 4 \mathrm{H}, J=6.0 \mathrm{~Hz}, 2 \times$ $\mathrm{ArH}), 7.09(\mathrm{~d}, 2 \mathrm{H} 2 \times \mathrm{ArH}), 7.15(\mathrm{~d}, 4 \mathrm{H}, J=6 \mathrm{~Hz}, 2 \times \mathrm{ArH}) 7.18(\mathrm{~d}, 2 \mathrm{H}, 2 \times \mathrm{ArH}),{ }^{13} \mathrm{C}$ NMR $\left(75 \mathrm{MHz}, \mathrm{CDCl}_{3}\right): \delta 27.1,50.5,50.9,51.6,104.8,116.3,118.5,121.1,122.7,129.6$, 135.8, 160.6, 162.9, 171.1 Anal. Calcd. for $\left(\mathrm{C}_{27} \mathrm{H}_{24} \mathrm{FNO}_{8}\right)$ : C, 63.65; H, 4.75; F, 3.73; N, 2.75; O, 25.12. Found. C,62.16; H, 4.77; F, 3.23,N, 2.70

\section{dimethyl1-(4-chlorophenyl)-5-(2,2-dimethyl-4,6-dioxo-1,3-dioxan-5-yl)-4-(4- fluorophenyl)-1H-pyrrole-2,3-dicarboxylate (5b)}

Characteristic: white crystalline solid M.P.: $166-170{ }^{\circ} \mathrm{C}$; IR (KBr): 3051, 2945, 1682, 1522 , 1513, 1294, 1174, 1072, 825,7 $53 \mathrm{~cm}^{-1}$; ${ }^{1} \mathrm{H}$ NMR (400 MHz, $\left.\mathrm{CDCl}_{3}\right): \delta 1.62(\mathrm{~s}, 6 \mathrm{H}$, $\left.\mathrm{C}\left(\mathrm{CH}_{3}\right)_{2}\right), 3.54\left(\mathrm{~s}, 6 \mathrm{H}\left(-\mathrm{COOCH}_{3}\right)\right), 5.51(\mathrm{~s}, 1 \mathrm{H}-\mathrm{CH}), 7.15(\mathrm{~d}, 4 \mathrm{H}, J=6.0 \mathrm{~Hz}, 2 \times \mathrm{ArH}), 7.19$ $(\mathrm{d}, 2 \mathrm{H} 2 \times \mathrm{ArH}), 7.19(\mathrm{~d}, 4 \mathrm{H}, J=6 \mathrm{~Hz}, 2 \times \mathrm{ArH}), 7.28(\mathrm{~d}, 2 \mathrm{H}, 2 \times \mathrm{ArH}),{ }^{13} \mathrm{C} \mathrm{NMR}(75 \mathrm{MHz}$, $\left.\mathrm{CDCl}_{3}\right): \delta 27.1,50.5,50.9,51.6,104.8,116.3,118.5,121.1,122.7,129.6,135.8,160.6$, 162.9, 171.1 Anal. Calcd. for $\left(\mathrm{C}_{26} \mathrm{H}_{21} \mathrm{ClFNO}_{8}\right): \mathrm{C}, 58.93 ; \mathrm{H}, 3.99 ; \mathrm{N}, 2.64 \mathrm{Cl}, 6.69 ; \mathrm{F}, 3.59 \mathrm{O}$, 24.15. Found. C, 58.53; H, 3.59; N, 2.24 Cl, 7.59; F, 3.17 O, 23.12.

\section{dimethyl5-(2,2-dimethyl-4,6-dioxo-1,3-dioxan-5-yl)-4-(4-fluorophenyl)-1-(4- methoxyphenyl)-1H-pyrrole-2,3-dicarboxylate (5c)}

Characteristic: white crystalline solid M.P.: $119-122{ }^{\circ} \mathrm{C}$; IR (KBr): 3022, 2825, 1671, 1625 , 1531, 1354, 1242, 1129, 753, $748 \mathrm{~cm}^{-1} ;{ }^{1} \mathrm{H}$ NMR (400 MHz, $\left.\mathrm{CDCl}_{3}\right): \delta 1.65(\mathrm{~s}, 6 \mathrm{H}$, $\left.\mathrm{C}\left(\mathrm{CH}_{3}\right)_{2}\right), 3.72\left(\mathrm{~s}, 3 \mathrm{H},-\mathrm{CH}_{3}\right), 3.82\left(\mathrm{~s}, 6 \mathrm{H}\left(-\mathrm{COOCH}_{3}\right)\right), 5.08(\mathrm{~s}, 1 \mathrm{H}-\mathrm{CH}), 7.37(\mathrm{~d}, 4 \mathrm{H}, J=$ $6.0 \mathrm{~Hz}, 2 \times \mathrm{ArH}), 7.39(\mathrm{~d}, 2 \mathrm{H} 2 \times \mathrm{ArH}), 7.39(\mathrm{~d}, 4 \mathrm{H}, J=6 \mathrm{~Hz}, 2 \mathrm{X} \mathrm{ArH}), 7.49(\mathrm{~d}, 2 \mathrm{H}, 2 \times$ $\mathrm{ArH}){ }^{13} \mathrm{C}$ NMR $\left(75 \mathrm{MHz}, \mathrm{CDCl}_{3}\right): \delta 27.1,51.5,52.6,105.8,108.3,117.5,121.6,133.7$, 134.6, 136.8, 141.6, 160.9, 162.1, 163.4, 177.2; Anal. Calcd. for $\left(\mathrm{C}_{27} \mathrm{H}_{24} \mathrm{FNO}_{9}\right): \mathrm{C}, 61.71 ; \mathrm{H}$, 4.60; F, 3.62; N, 2.67; O, 27.40 Found. C, 60.93; H, 3.99; N, 2.64; F, 3.59 O, 26.15.

dimethyl5-(2,2-dimethyl-4,6-dioxo-1,3-dioxan-5-yl)-1,4-bis(4-fluorophenyl)-1H-pyrrole2,3-dicarboxylate (5d)

Characteristic: white crystalline solid M.P.: $162-164{ }^{\circ} \mathrm{C}$; IR $(\mathrm{KBr}): 3036,2844,1639,1622$, 1572, 1312, 1247, 1197, 824, $763 \mathrm{~cm}^{-1} ;{ }^{1} \mathrm{H}$ NMR (400 MHz, $\left.\mathrm{CDCl}_{3}\right): \delta 1.67(\mathrm{~s}, 6 \mathrm{H}$, $\left.\mathrm{C}\left(\mathrm{CH}_{3}\right)_{2}\right), 3.90\left(\mathrm{~s}, 6 \mathrm{H}\left(-\mathrm{COOCH}_{3}\right)\right), 4.91(\mathrm{~s}, 1 \mathrm{H}-\mathrm{CH}), 7.24(\mathrm{~d}, 4 \mathrm{H}, J=6.0 \mathrm{~Hz}, 2 \times \mathrm{ArH}), 7.30$ $(\mathrm{d}, 2 \mathrm{H} 2 \times \mathrm{ArH}), 7.36(\mathrm{~d}, 4 \mathrm{H}, J=6 \mathrm{~Hz}, 2 \times \mathrm{ArH}), 7.60(\mathrm{~d}, 2 \mathrm{H}, 2 \times \mathrm{ArH}),{ }^{13} \mathrm{C} \mathrm{NMR}(75$ $\mathrm{MHz}, \mathrm{CDCl}_{3}$ ): $\delta$ 29.1, 51.5, 104.8, 116.0, 116.5, 121.7, 123.6, 128.4, 130.2, 160.1, 162.9, 165.7, 172.3 Anal. Calcd. for $\left(\mathrm{C}_{26} \mathrm{H}_{21} \mathrm{~F}_{2} \mathrm{NO}_{8}\right): \mathrm{C}, 60.82 ; \mathrm{H}, 4.12 ; \mathrm{F}, 7.40 ; \mathrm{N}, 2.73 ; \mathrm{O}, 24.9$ Found. C, 60.53; H, 4.99; N, 2.64; F, 7.59 O, 24.15.

dimethyl5-(2,2-dimethyl-4,6-dioxo-1,3-dioxan-5-yl)-4-(4-fluorophenyl)-1-(4nitrophenyl)-1H-pyrrole-2,3-dicarboxylate (5e)

Characteristic: white crystalline solid M.P.: $146-150{ }^{\circ} \mathrm{C}$; IR $(\mathrm{KBr}): 3032,2845,1691,1622$, 1528, 1394, 1274, 1179, $753 \mathrm{~cm}^{-1} ;{ }^{1} \mathrm{H}$ NMR (400 MHz, $\left.\mathrm{CDCl}_{3}\right): \delta 1.67\left(\mathrm{~s}, 6 \mathrm{H}, \mathrm{C}\left(\mathrm{CH}_{3}\right)_{2}\right)$, $4.01\left(\mathrm{~s}, 6 \mathrm{H}\left(-\mathrm{COOCH}_{3}\right)\right), 4.41(\mathrm{~s}, 1 \mathrm{H}-\mathrm{CH}), 7.30(\mathrm{~d}, 4 \mathrm{H}, J=6.0 \mathrm{~Hz}, 2 \times \mathrm{ArH}), 7.39(\mathrm{~d}, 2 \mathrm{H} 2 \times$ $\mathrm{ArH}), 7.51(\mathrm{~d}, 4 \mathrm{H}, J=6 \mathrm{~Hz}, 2 \times \mathrm{ArH}), 7.60(\mathrm{~d}, 2 \mathrm{H}, 2 \times \mathrm{ArH}),{ }^{13} \mathrm{CNMR}\left(75 \mathrm{MHz}, \mathrm{CDCl}_{3}\right)$ : 
829.2, 45.3, 47.1, 101.4, 116.2, 117.4, 120.0, 122.4, 123.6, 128.4, 132.1, 134.5, 141.7, 162.3, 168.1, 172.2 Anal. Calcd. for $\left(\mathrm{C}_{26} \mathrm{H}_{21} \mathrm{BrFNO}_{8}\right)$ : C, 57.78; H, 3.92; F, 3.52; $\mathrm{Br} 2.41 ; \mathrm{N}, 5.18$; O, 29.60 Found. C, 58.93; H, 3.79; N, 5.64; F, 3.34; Br, 2.72; O, 28.15.

\section{dimethyl1-(4-bromophenyl)-5-(2,2-dimethyl-4,6-dioxo-1,3-dioxan-5-yl)-4-(4- fluorophenyl)-1H-pyrrole-2,3-dicarboxylate (5f)}

Characteristic: white crystalline solid M.P.: $146-150{ }^{\circ} \mathrm{C}$; IR (KBr): 3022, 2835, 1674, 1617 , 1546, 1214, 1201, 1165, 1023, 865, $753 \mathrm{~cm}^{-1} ;{ }^{1} \mathrm{H}$ NMR $\left(400 \mathrm{MHz}, \mathrm{CDCl}_{3}\right): \delta 1.67(\mathrm{~s}, 6 \mathrm{H}$, $\left.\mathrm{C}\left(\mathrm{CH}_{3}\right)_{2}\right), 3.91\left(\mathrm{~s}, 6 \mathrm{H}\left(-\mathrm{COOCH}_{3}\right)\right), 5.06(\mathrm{~s}, 1 \mathrm{H}-\mathrm{CH}), 7.32(\mathrm{~d}, 4 \mathrm{H}, J=6.0 \mathrm{~Hz}, 2 \times \mathrm{ArH}), 7.37$ $(\mathrm{d}, 2 \mathrm{H} 2 \times \mathrm{ArH}), 7.68(\mathrm{~d}, 4 \mathrm{H}, J=6 \mathrm{~Hz}, 2 \times \mathrm{ArH}), 8.18(\mathrm{~d}, 2 \mathrm{H}, 2 \times \mathrm{ArH}),{ }^{13} \mathrm{CNMR}(75 \mathrm{MHz}$, $\mathrm{CDCl}_{3}$ ): $\delta 28.1,51.3,104.5,116.0,121.9,124.5,127.1,130.8,134.1,144.7,160.1,162.9$, 173.1; Anal. Calcd. for $\left(\mathrm{C}_{26} \mathrm{H}_{21} \mathrm{FN}_{2} \mathrm{O}_{10}\right)$ : C, 54.37; H, 3.69; Br, 13.91; F, 3.31; N, 2.44; O, 22.29 Found. C, 54.93; H, 3.99; N, 2.64; Br, 13.52,F, 3.59 O, 22.75.

\section{dimethyl,4-(4-fluorophenyl)-1-(p-tolyl)-5-(2,4,6-trioxohexahydropyrimidin-5-yl)-1H-} pyrrole-2,3-dicarboxylate (5g)

Characteristic: white crystalline solid (b) M.P.: $155-161^{\circ} \mathrm{C}$; IR $(\mathrm{KBr})$ : 3027, 2835, 1671, $1615,1548,1364,1224,1079,743 \mathrm{~cm}^{-1} ;{ }^{1} \mathrm{H}$ NMR (400 MHz, $\left.\mathrm{CDCl}_{3}\right): \delta 2.27\left(\mathrm{~s}, 3 \mathrm{H},-\mathrm{CH}_{3}\right)$, 3.62-3.64 (s, 6H(-COOCH 3$)), 4.66(\mathrm{~s}, 1 \mathrm{H}-\mathrm{CH}), 7.08(\mathrm{~d}, 4 \mathrm{H}, J=6 \mathrm{~Hz}, 2 \times \mathrm{ArH}), 7.15(\mathrm{~d}, 2 \mathrm{H}$ $2 \times \mathrm{ArH}), 7.24(\mathrm{~d}, 4 \mathrm{H}, J=6 \mathrm{~Hz}, 2 \times \mathrm{ArH}), 7.39(\mathrm{~d}, 2 \mathrm{H}, 2 \times \mathrm{ArH}), 10.03(1 \mathrm{H}, \mathrm{s}$, exchangeable, $\mathrm{NH}) .{ }^{13} \mathrm{C}$ NMR $\left(75 \mathrm{MHz}, \mathrm{CDCl}_{3}\right): \delta 27.1,50.5,50.9,51.6,104.8,116.3$, 118.5, 121.1, 122.7, 129.6, 135.8, 160.6, 162.9, 171.1;Anal. Calcd. for $\left(\mathrm{C}_{25} \mathrm{H}_{20} \mathrm{FN}_{3} \mathrm{O}_{7}\right): \mathrm{C}$, 57.78; H, 3.92; F, 3.52; N, 5.18; O, 29.60 Found. C, 57.36; H, 3.64; N, 5.58; ,F, $3.12 \mathrm{O}$, 29.15.

dimethyl1-(4-chlorophenyl)-4-(4-fluorophenyl)-5-(2,4,6-trioxohexahydropyrimidin-5yl)-1H-pyrrole-2,3-dicarboxylate (5h)

Characteristic: white crystalline solid (b) M.P.: 159-163 ${ }^{\circ} \mathrm{C}$; IR (KBr): 3025, 2935, 1691, $1625,1542,1354,1324,1201,1069,864,743 \mathrm{~cm}^{-1} ;{ }^{1} \mathrm{H}$ NMR $\left(400 \mathrm{MHz}, \mathrm{CDCl}_{3}\right): \delta 3.73(\mathrm{~s}$, $\left.6 \mathrm{H}\left(-\mathrm{COOCH}_{3}\right)\right), 5.85(\mathrm{~s}, 1 \mathrm{H},-\mathrm{CH}), 7.18(\mathrm{~d}, 4 \mathrm{H}, J=6 \mathrm{~Hz}, 2 \times \mathrm{ArH}), 7.25(\mathrm{~d}, 2 \mathrm{H} 2 \times \mathrm{ArH})$, 7.34 (d, 4H, $J=6 \mathrm{~Hz}, 2 \times \mathrm{ArH}), 7.49$ (d, 2H, $2 \times \mathrm{ArH}), 10.13(1 \mathrm{H}, \mathrm{s}$, exchangeable, NH). ${ }^{13} \mathrm{CNMR}\left(75 \mathrm{MHz}, \mathrm{CDCl}_{3}\right)$ : $\delta 51.3,116.2,120.7,121.8,126.1,128.3,129.4,131.1,162.9$, 163.3, Anal. Calcd. for $\left(\mathrm{C}_{24} \mathrm{H}_{17} \mathrm{ClFN}_{3} \mathrm{O}_{7}\right): \mathrm{C}, 56.10 ; \mathrm{H}, 3.33 ; \mathrm{Cl}, 6.90 ; \mathrm{F}, 3.70 ; \mathrm{N}, 8.18$; O, 21.80 Found. C, 56.93; H, 3.42; Cl, 6.75N, 2.64; F, 3.59 O, 24.15.

dimethyl4-(4-fluorophenyl)-1-(4-methoxyphenyl)-5-(2,4,6-trioxohexahydropyrimidin-5yl)-1H-pyrrole-2,3-dicarboxylate (5i)

Characteristic: white crystalline solid (b) M.P.: 147-151 ${ }^{\circ} \mathrm{C}$; IR (KBr): 3037, 2935, 1699, $1625,1568,1354,1224,1102,1049,875,743 \mathrm{~cm}^{-1} ;{ }^{1} \mathrm{H}$ NMR (400 MHz, $\left.\mathrm{CDCl}_{3}\right): \delta 3.38(\mathrm{~s}$, $\left.3 \mathrm{H},-\mathrm{CH}_{3}\right), 3.91\left(\mathrm{~s}, 6 \mathrm{H}\left(-\mathrm{COOCH}_{3}\right)\right), 4.96(\mathrm{~s}, 1 \mathrm{H}-\mathrm{CH}), 6.99(\mathrm{~d}, 4 \mathrm{H}, J=6 \mathrm{~Hz}, 2 \times \mathrm{ArH}), 7.15$ $(\mathrm{d}, 2 \mathrm{H} 2 \times \mathrm{ArH}), 7.24(\mathrm{~d}, 4 \mathrm{H}, J=6 \mathrm{~Hz}, 2 \times \mathrm{ArH}), 7.31(\mathrm{~d}, 2 \mathrm{H}, 2 \times \mathrm{ArH}), 10.02(1 \mathrm{H}, \mathrm{s}$, exchangeable, $\mathrm{NH}) .{ }^{13} \mathrm{CNMR}\left(75 \mathrm{MHz}, \mathrm{CDCl}_{3}\right): \delta 51.5,55.8,114.9,116.2,120.3,121.9$, 126.5, 127.2, 130.3, 141.0, 159.3, 162.4, 169.8; Anal. Calcd. for $\left(\mathrm{C}_{25} \mathrm{H}_{20} \mathrm{FN}_{3} \mathrm{O}_{8}\right): \mathrm{C}, 58.94 ; \mathrm{H}$, 3.96; F, 3.73; N, 8.25; O, 25.12; Found. C, 58.93; H, 3.99; N, 2.64; F, 3.59 O, 24.15.

dimethyl1,4-bis(4-fluorophenyl)-5-(2,4,6-trioxohexahydropyrimidin-5-yl)-1H-pyrrole2,3-dicarboxylate $(5 \mathbf{j})$

Characteristic: white crystalline solid (b) M.P.: 171-174 ${ }^{\circ} \mathrm{C}$; IR (KBr): 3036, 2844, 1689, 
1622, 1572, 1312, 1247, 1197, 824, $763 \mathrm{~cm}^{-1} ;{ }^{1} \mathrm{H}$ NMR (400 MHz, $\left.\mathrm{CDCl}_{3}\right): 3.72$ (s, 6H($\left.\left.\mathrm{COOCH}_{3}\right)\right), 4.86(\mathrm{~s}, 1 \mathrm{H}-\mathrm{CH}), 7.18(\mathrm{~d}, 4 \mathrm{H}, J=6 \mathrm{~Hz}, 2 \times \mathrm{ArH}), 7.25(\mathrm{~d}, 2 \mathrm{H} 2 \times \mathrm{ArH}), 7.34(\mathrm{~d}$, $4 \mathrm{H}, J=6 \mathrm{~Hz}, 2 \times \mathrm{ArH}), 7.39(\mathrm{~d}, 2 \mathrm{H}, 2 \times \mathrm{ArH}), 10.13(2 \mathrm{H}, \mathrm{s}$, exchangeable, $\mathrm{NH}) .{ }^{13} \mathrm{C} \mathrm{NMR}$ $\left(75 \mathrm{MHz}, \mathrm{CDCl}_{3}\right): \delta 50.5,114.8,116.3,120.5,121.1,122.7,129.6,135.8,141.5,159.2$, 160.6, 162.9, 171.1; Anal. Calcd. for $\left(\mathrm{C}_{24} \mathrm{H}_{17} \mathrm{~F}_{2} \mathrm{~N}_{3} \mathrm{O}_{7}\right)$ : C, 57.95; H, 3.44; F, 7.64; N, 8.45; O, 22.52.Found. C, 58.93; H,3.99; F, 3.59 N, 2.64O, 24.15.

\section{dimethyl1-(4-bromophenyl)-4-(4-fluorophenyl)-5-(2,4,6-trioxohexahydropyrimidin-5-} yl)-1H-pyrrole-2,3-dicarboxylate (5k)

Characteristic: white crystalline solid (b) M.P.: 159-164 ${ }^{\circ} \mathrm{C}$; IR (KBr): 3077, 2854, 1686, $1614,1554,1394,1221,1154,1032,1019,864,723 \mathrm{~cm}^{-1} ;{ }^{1} \mathrm{H}$ NMR (400 MHz, $\left.\mathrm{CDCl}_{3}\right): \delta 3.81$ $\left(\mathrm{s}, 6 \mathrm{H}\left(-\mathrm{COOCH}_{3}\right)\right), 4.87(\mathrm{~s}, 1 \mathrm{H}-\mathrm{CH}), 7.17(\mathrm{~d}, 4 \mathrm{H}, J=6 \mathrm{~Hz}, 2 \times \mathrm{ArH}), 7.23(\mathrm{~d}, 2 \mathrm{H} 2 \times \mathrm{ArH})$, $7.32(\mathrm{~d}, 4 \mathrm{H}, J=6 \mathrm{~Hz}, 2 \times \mathrm{ArH}), 7.49(\mathrm{~d}, 2 \mathrm{H}, 2 \times \mathrm{ArH}), 10.0(1 \mathrm{H}, \mathrm{s}$, exchangeable, $\mathrm{NH}) .{ }^{13} \mathrm{C}$ $\operatorname{NMR}\left(75 \mathrm{MHz}, \mathrm{CDCl}_{3}\right): \delta 51.6,114.9,116.3,118.5,121.4,122.4,128.6,133.8,142.3,153.2$, 160.6, 162.9, 170.1; Anal. Calcd. for $\left(\mathrm{C}_{24} \mathrm{H}_{17} \mathrm{BrFN}_{3} \mathrm{O}_{7}\right)$ : C, 51.63; H, 3.07; Br, 14.31; F, 3.40; N, 7.53; O, 20.06Found. C, 58.93; H, 3.99; Br, 14.31; F, 3.59 N, 2.64O, 24.15.

\section{CONCLUSION}

In summary, we have developed an exceedingly simple, mild, clean and expeditious synthetic protocol for penta substituted pyrroles derivatives. Remarkable advantages of this synthetic strategy over the others are (i) high yields, (ii) no need of acid/base catalyst and solvent, (iii) decreased reaction times, (iv) simplified work-up procedure, and (v) ambient reaction temperature

\section{ACKNOWLEDGEMENT}

We are thankful to FIST-DST and SAP-UGC for the irgenerous financial and instrumentation support. Special thanks are due to "National Facility for Drug Discovery (NFDD) through New Chemical Entities (NCE's) Development \& Instrumentation Support to Small Manufacturing Pharma Enterprises" Programme under Drug \& Pharma Research Support (DPRS) jointly funded by Department of Science \& Technology, New Delhi, Government of Gujarat Industries Commissioner rate \& Saurashtra University, Rajkot, Gujarat, India

\section{References}

[1] B. Thompson, J. Montgomery, Org. Lett. 13 (2011) 3289.

[2] B. Trost, A. Gutierrez, R. Livingston, Org. Lett. 11 (2009) 2539.

[3] N. Ganguly, S. Roy, P. Mondal, R. Saha, Tetrahedron Lett. 53 (2012) 7067.

[4] L. Wen, Y. Shi, G. Liu, Org. Chem. 77 (2012) 4252.

[5] D. Hong, Y. Zhu, Y. Li, X.Lin, P. Lu ,Y. Wang, Org. Lett. 13 (2011) 4668.

[6] B. Lee, M. Clothier, F. Dutton, G. Conder, S. Johnson, Bioorg. Med.Chem. Lett. 8 (1998) 3317.

[7] J. Jung, Y. Jung, O. Park, Synth. Commun. 31(2001) 1195.

[8] S. Hesse G. Kirsch, Tetrahedron Lett. 43 (2002) 1213. 
[9] J. Jung, J. Lee, S. Oh, J. Lee, O. Park, Bioorg. Med. Chem. Lett. 14 (2004) 5527.

[10] G. Melagraki, A. Afantitis, O. Igglessi-Markopoulou, A. Detsi, M. Koufaki, C. Kontogiorgis, D. J. Hadjipavlou-Litina, Eur. J. Med. Chem. 44 (2009) 3020. 